\title{
Relación entre obesidad y enfermedad periodontal: revisión de la literatura
}

\author{
Jorge Girano Castaños*1,a ; José Robello Malatto ${ }^{1, b}$
}

RESUMEN

La obesidad es una enfermedad que va en aumento sin distinguir género o edad, y en numerosas ocasiones es un factor de riesgo para otras dolencias sistémicas. Es considerada un cuadro progresivo que afecta la salud general y la calidad de vida del individuo. Según diversos estudios, la obesidad se puede relacionar a enfermedades de la cavidad oral como resultado de una alteración fisiológica del organismo que tiene efecto en los tejidos blandos de la boca, lo que se evidencia en signos clínicos de inflamación en el periodonto.

El objetivo principal de este trabajo es evaluar la relación entre obesidad y enfermedad periodontal. Para ello se realizó una búsqueda de artículos que determinen el nexo entre ambas enfermedades. La presente revisión presenta diversos estudios que muestran una posible relación entre ambas enfermedades. La intervención de mediadores inflamatorios en la obesidad podría tener una repercusión a nivel periodontal.

Se concluye, con base en la evidencia disponible, que es probable que exista una relación entre enfermedad periodontal y obesidad.

Palabras clave: Obesidad; Índice de masa corporal; Síndrome metabólico; Enfermedades periodontales (Fuente: DeCS BIREME).

\section{Relationship between obesity and periodontal disease: a literature review}

\begin{abstract}
Obesity is a disease whose prevalence is on the rise. It does not discriminate in terms of gender or age, and is often considered a risk factor for different systemic diseases. It is also considered a progressive disease that affects not only the general health but also the quality of life of individuals. Several studies have reported that obesity may also be related to oral diseases as a result of a physiological alteration of the body that affects the soft tissues of the oral cavity, which is evidenced by clinical signs of periodontal inflammation. The main objective of this review article is to evaluate the relationship between obesity and periodontal disease. To this end, previously published studies that determine the relationship between both diseases were searched. This review presents several articles that show a possible relationship between both diseases. The intervention of inflammatory mediators in obesity could have a periodontal impact. Based on the available evidence, it is concluded that there is a possible relationship between periodontal disease and obesity.
\end{abstract}

Keywords: Obesity; Body mass index; Metabolic syndrome; Periodontal diseases (Source: MeSH NLM).

\footnotetext{
1 Universidad de San Martín de Porres, Facultad de Odontología. Lima, Perú.

a Docente.

b Director del Centro Odontológico.

* Autor corresponsal
} 


\section{INTRODUCCIÓN}

La Organización Mundial de la Salud (OMS) define a la obesidad como una acumulación excesiva de grasa que puede llegar a ser perjudicial para la salud. Este cuadro ha duplicado su frecuencia en la última década. En 2008, 1400 millones de adultos tenían sobrepeso. Según datos estadísticos, 200 millones de hombres y cerca de 300 millones de mujeres padecen de obesidad ${ }^{(1)}$. El Instituto Nacional de Salud de los Estados Unidos de América, a través de la Encuesta Nacional De Salud y Nutrición del 2012 (National Health and Nutrition Examination Survey, NHANES, por su sigla en inglés), reportó una prevalencia del $35,7 \%$ de obesidad y sobrepeso en adultos (78 millones de personas aproximadamente) y una prevalencia de $16,9 \%$ en niños y adolescentes (casi 12,5 millones) (2). En el Perú, el sobrepeso y la obesidad constituyen un problema de salud pública. Alrededor del $62,3 \%$ de adultos jóvenes sufren de sobrepeso y obesidad (3).

Por otra parte, la enfermedad periodontal es la causa principal de pérdida de dientes en adultos; afecta las estructuras de soporte del diente, las encías, la membrana periodontal y el hueso alveolar. En el estado más avanzado, la periodontitis es el resultado de una extensión del proceso inflamatorio iniciado desde la encía hacia el tejido periodontal de soporte ${ }^{(4)}$.

De acuerdo a la OMS, las enfermedades periodontales graves afectan del 5 al $20 \%$ de los adultos de edad madura en el mundo, con una incidencia que varía según la ubicación geográfica ${ }^{(5)}$. Según el Ministerio de Salud del Perú (Minsa), la prevalencia de la enfermedad periodontal en nuestro país es de $85 \%$. Por tanto, en el 2005, el Gobierno Peruano emitió una Resolución Ministerial (n. ${ }^{\circ} 538$ - 2005/MINSA) que describe la situación de la salud bucal en nuestra población como crítica ${ }^{(6)}$.

Estudios recientes señalan el importante papel de la nutrición en el ámbito de la enfermedad periodontal y muestran que la obesidad podría ser un factor de riesgo potencial para desarrollar este cuadro. La obesidad tiene impacto en los parámetros metabólicos e inmunes y a través de ellos puede aumentar la susceptibilidad del huésped a la enfermedad periodontal ${ }^{(7)}$. Por ello, el objetivo de esta revisión es evaluar, mediante la evidencia disponible, la posible relación entre obesidad y enfermedad periodontal.

\section{Estrategia de búsqueda}

Este trabajo es una revisión bibliográfica sobre la relación entre obesidad y enfermedad periodontal. Se realizó la búsqueda de información en bases de datos vía web (Pubmed, Sciencedirect, Ebsco) con las siguientes palabras clave: obesidad, índice de masa corporal (IMC), síndrome metabólico (SM) yenfermedad periodontal. Seseleccionaron artículos descriptivos y analíticos que determinaran la posible relación entre ambas enfermedades y se analizaron 50, aproximadamente. Luego, de acuerdo a los objetivos y diseños de cada estudio, se seleccionaron 35 de los que se escogieron solo 15 que cumplían los criterios de inclusión y que se revisaron para esta investigación.

\section{Obesidad}

La obesidad es una enfermedad compleja y multifactorial que se desarrolla a partir de una interacción del genotipo y el medio ambiente, y se caracteriza por acumulación anormal de grasa o hipertrofia general del tejido adiposo en el cuerpo ${ }^{(8)}$. Se considera que el tejido adiposo es un tipo de tejido conectivo compuesto por adipocitos que produce altos niveles de citoquinas y hormonas como las adipocinas que, a su vez, producen leptina (que regula el gasto de energía y metabolismo) y adiponectina (reguladora de glucosa, presión sanguínea, catabolismo de los ácidos grasos, entre otros) ${ }^{(9)}$. La obesidad es considerada como un factor de riesgo para diversas enfermedades sistémicas como hipertensión, diabetes mellitus, arteriosclerosis, enfermedades cardiovasculares, entre otros ${ }^{(10)}$. La OMS establece que existe obesidad si el índice de masa corporal (IMC) es $\geq 30 \mathrm{~kg} / \mathrm{m}^{2}$. El IMC de una persona se obtiene dividiendo su peso (en kilogramos) entre el cuadrado de su altura (en metros). Además, un perímetro abdominal $\geq 102$ $\mathrm{cm}$ en hombres, $\mathrm{y} \geq 88 \mathrm{~cm}$ en mujeres se considera como signo de obesidad ${ }^{(11)}$.

\section{Enfermedad periodontal}

La enfermedad periodontal (EP) es la principal causa de pérdida de dientes en adultos. Es un cuadro que afecta las estructuras de soporte del diente, las encías, la membrana periodontal y el hueso alveolar. La periodontitis es el resultado de una extensión del proceso inflamatorio iniciado desde la encía hacia el tejido periodontal de soporte. Se caracteriza por la inflamación de las encías, la presencia de placa subgingival bacterias patógenas, la pérdida de inserción clínica con formación de bolsas debido a la destrucción del ligamento periodontal y la pérdida del hueso adyacente ${ }^{(12)}$.

La periodontitis depende de las respuestas del huésped a patógenos periodontales. Los neutrófilos y macrófagos que aparecen al inicio del proceso ocasionan la liberación de citoquinas como el factor de necrosis tumoral alfa (FNT-a), la interleucina-1 (IL-1), y las prostaglandinas (12). En el proceso inflamatorio, la IL-1 estimula los fibroblastos y se secretan las metaloproteinasas de la matriz (MMP) que son responsables del aumento de la degradación del colágeno (la colagenasa es la MMP más importante). Además, el FNT-a aumenta la actividad de los osteoclastos, lo que resulta en resorción ósea ${ }^{(13)}$.

Relación entre la obesidad y la enfermedad periodontal Por largo tiempo, el tejido adiposo fue considerado como 
un sistema inerte que almacenaba los triglicéridos. Sin embargo, hoy se sabe que es un órgano complejo que secreta numerosos factores inmunomoduladores y desempeña un papel importante en la regulación metabólica y biología vascular. Las células adiposas, que incluyen adipocitos y macrófagos, secretan más de 50 moléculas bioactivas, conocidas como adipocinas. Algunas de ellas actúan de manera local y otras se liberan en la circulación sistémica y funcionan como moléculas de señalización en el hígado, el músculo y el endotelio ${ }^{(14)}$. Las adipocinas desempeñan funciones diferentes como hormonas o proteínas (leptina y adiponectina, por ejemplo). La adiponectina modula un número de procesos metabólicos como la regulación de la glucosa, la presión sanguínea, el catabolismo de los ácidos grasos y tiene asociaciones inversas con marcadores séricos de la inflamación (antiinflamatorios) ${ }^{(15)}$.

Los niveles bajos de adiponectina se asocian con un mayor riesgo de enfermedad de la arteria coronaria y otras características del síndrome metabólico. Por otra parte, en la obesidad existe un estado inflamatorio que incrementa la producción de FNT- $a$, leptina, IL-1 e IL- 6 secretadas por los adipocitos y macrófagos del tejido adiposo blanco ${ }^{(16,17)}$.

Estas citocinas son importantes en el desarrollo y la progresión de la enfermedad periodontal porque su liberación está estrechamente relacionada con una mayor susceptibilidad a la infección bacteriana, causada por una alteración en la respuesta inmune del huésped ${ }^{(18,19)}$. El FNT-a estimula la reabsorción ósea, la degradación del colágeno y la activación de células endoteliales (VCAM/ ICAM), también aumenta la producción de IL-8 y la expresión de MCP-1, además, incrementa la resistencia a la insulina e induce proteína C-reactiva ${ }^{(20)}$. Además, el FNT-a es un potente inhibidor de la adiponectina, una adipocina con propiedades antiinflamatorias importantes (21) que le permiten actual como un modulador endógeno en las enfermedades relacionadas con la obesidad.

\section{Antecedentes de la relación entre obesidad y enfermedad periodontal}

Existen numerosos estudios acerca de la relación entre obesidad y enfermedad periodontal. En nuestra investigación incluimos los siguientes:

Vecchia et al. (2005) (22) evaluaron la asociación del sobrepeso y la obesidad con la periodontitis en adultos brasileños y encontraron una correlación positiva entre el índice IMC y la aparición de la periodontitis $(p=0,05)$. $\mathrm{El}$ análisis multivariado mostró que las mujeres obesas tenían significativamente más probabilidades (OR 2 , 1 : IC $95 \%: 1,1-3,9)$ de tener periodontitis en comparación con las mujeres con peso normal. Un análisis separado de los no fumadores mostró que las mujeres obesas tenían casi 3,4 veces más probabilidades de tener periodontitis que el grupo con IMC normal. El estudio concluye que la obesidad, pero no el sobrepeso, se asocia significativamente con la periodontitis en adultos, y que fumar puede atenuar la asociación de periodontitis con la obesidad.

En 2008, Taghi Chitzasi et al. ${ }^{23)}$ investigaron la relación entre enfermedad periodontal, proteína C-reactiva (PCR) y obesidad. Los resultados revelaron una diferencia estadísticamente significativa entre todas las variables analizadas en los grupos de prueba y control $(p=0,05)$. Los sujetos de prueba se clasificaron en dos subgrupos (participantes con $P C R \geq 3 \mathrm{mg} / \mathrm{l}$ y con $P C R \leq 3 \mathrm{mg} /$ ).

Los OR más altos corresponden a las mujeres con mayor circunferencia de cintura (WC por su sigla en inglés) $(O R=6,4)$ (IC $95 \%: 1,18$ a 35,2) $(p=0,02)$, y en los hombres a la obesidad $(\mathrm{OR}=4,8 ; \mathrm{IC} 95$ \%: 0,65 a 35,19; $p=0,05)$. Al considerar la correlación entre la obesidad, el sobrepeso, WC y la PCR con la profundidad de sondaje y pérdida de inserción, se observa que la obesidad presenta la asociación más alta $(r=1$; $p=0,00)$ y el sobrepeso la más baja $(r=0,4 ; p=0,07)$. El resultado del estudio indica que la enfermedad periodontal está correlacionada con elevación del PCR y las enfermedades asociadas con la obesidad.

D'Aiuto et al. (2008) (24) determinaron la asociación entre síndrome metabólico y periodontitis. El síndrome metabólico se definió como la concurrencia de hipertensión, obesidad, resistencia a la insulina y un perfil lipídico aterogénicos como hipertrigliceridemia y colesterol bajo en lipoproteínas de alta densidad $(\mathrm{HDL})$. Los resultados mostraron que la prevalencia del síndrome metabólico fue de 18 \% (IC $95 \% 16$ a 19) en individuos sin periodontitis; 34 \% (IC $95 \%$ 29-38) en casos de periodontitis moderada, y $37 \%$ (IC $95 \%$ 28-48) en la periodontitis severa. Después de ajustar los factores de confusión, los pacientes mayores de 45 años que sufren de periodontitis severas eran 2,31 veces más propensos a presentar síndrome metabólico (IC 95\%: 1,13 a 4,73 ), en comparación a los individuos que no la sufren. El estudio concluyó que la periodontitis severa se asocia con el síndrome metabólico en individuos de mediana edad.

Ylostalo et al. (2008) ${ }^{(25)}$ examinaron la relación entre el peso corporal y la infección periodontal. Los resultados mostraron una asociación entre el peso corporal y la infección periodontal en la población no diabética de no fumadores de 30-49 años de edad. Sin embargo, para el autor se necesita investigación adicional para determinar la naturaleza de esta relación.

Khader et al. (2008) (26) realizaron un estudio para evaluar la distribución de los parámetros periodontales entre los pacientes con síndrome metabólico (SM) y sujetos sin SM en el norte de Jordania. Los pacientes fueron diagnosticados con SM según los criterios del Programa Nacional de Educación sobre el Colesterol (ATP III, por su sigla en inglés). Los criterios ATP III para SM se cumplen 
si un individuo tenía 3 o más de los siguientes cuadros: obesidad, glucosa alta en ayunas, presión arterial alta y/o hipertrigliceridemia. Todos los pacientes con SM eran obesos. Los autores concluyen que los pacientes con SM presentan una enfermedad periodontal más grave y extensa en comparación con los sujetos sin SM

Khader et al. (2009) (7) determinaron la relación entre periodontitis y sobrepeso/obesidad entre los jordanos. En el estudio participaron 340 personas de entre 18 y 70 años que acudían al centro médico de la Universidad de Ciencia y Tecnología de Jordania. Los resultados mostraron que la obesidad definida por el índice de masa corporal (IMC) [(OR) 2,9, (IC) $95 \%: 1,3-6,1]$, valor de circunferencia abdominal alto (WC) [(OR 2,1; IC $95 \%: 1,2-3,7)]$, y un elevado porcentaje de grasa [(OR 1,8; IC $95 \%$ : 1,03-3,3)] permaneció significativamente asociada con mayores probabilidades de periodontitis. El estudio concluyó que la obesidad medida por el IMC y el valor alto de la circunferencia de la cintura se asociaron significativamente con el aumento de probabilidad de tener periodontitis.

Saxlin et al. (2010) (27) realizaron una investigación longitudinal de 4 años para investigar la asociación entre el peso corporal y la infección periodontal. Este trabajo se basó en una subpoblación de la Encuesta de Salud 2000 que incluyó sujetos dentados entre 30 y 59 años y que habían participado en el estudio de seguimiento sobre la salud bucal de los adultos finlandeses, aproximadamente 4 años después $(n=5396)$ El resultado del estudio muestra que el peso corporal era solo débilmente, pero no estadísticamente, significativo asociado con el número de dientes con bolsas periodontales profundas (periodontitis) [RR 1,1 (IC $95 \%$ 0,8-1,7)]. El estudio concluyó que los resultados del seguimiento no aportan pruebas de que el sobrepeso y la obesidad pueden ser considerados factores de riesgo importantes en la patogénesis de la infección periodontal. En ese mismo año, Chaffee et al. ${ }^{(4)}$ elaboraron un metaanálisis con la información disponible. Esta investigación trató de compilar de forma sistemática, la evidencia de una relación entre la enfermedad periodontal y la obesidad a partir de estudios epidemiológicos. Se seleccionaron varios artículos que cumplían con los criterios de inclusión del estudio para su análisis. Encontraron una asociación entre la enfermedad periodontal y la obesidad [OR 1,35 (IC 95\% 1,23-1,47)] y, además, la evidencia de una relación más fuerte en adultos jóvenes y mujeres. La revisión concluye que es probable que la prevalencia de enfermedad periodontal sea mayor entre los pacientes obesos, aunque no hay ninguna evidencia actual para recomendar un cambio en la planificación del tratamiento.

Otro estudio realizado por Saxlin et al. en 2011 ${ }^{(28)}$ investigó el rol de la infección periodontal en la obesidad en la población adulta. Se encontró que el número de dientes con bolsas periodontales profundas podría estar asociado con el IMC de una manera dosis-respuesta entre la población total del estudio. La investigación estableció que la infección periodontal parece asociarse con la obesidad, aunque no existen indicios de la causalidad. Concluyen que se necesitan más estudios para aclarar el posible papel de la infección periodontal en la obesidad.

En 2013, Palle et al. (29) realizaron un estudio transversal en pacientes con enfermedades cardiovasculares para correlacionar la obesidad (IMC y WC) y los parámetros de la enfermedad periodontal. Se examinaron a 201 pacientes. Los sujetos que tenían antecedentes de enfermedades cardiovasculares y en tratamiento fueron incluidos en el estudio. Se emplearon 3 parámetros periodontales (profundidad de sondeo, nivel de inserción clínica e índice de higiene bucal $(\mathrm{OHI})$ y dos indicadores de obesidad (IMC y WC). La influencia del IMC y otras variables de confusión en la severidad de la periodontitis. Los resultados mostraron una asociación significativa ( $p<0,005$, en cada caso) de la gravedad de la periodontitis con las lipoproteínas de baja densidad (LDL), los triglicéridos (TGL) y el colesterol. También existe asociación significativa $(p<0,001)$ entre la gravedad de la periodontitis y el IMC, el índice de higiene oral $(\mathrm{OHI}$, por su sigla en inglés) y la circunferencia de cintura. Los autores concluyeron que la obesidad se ha implicado como un factor de riesgo para varias afecciones, incluidas las enfermedades cardiovasculares, diabetes, etc. La relación entre las medidas de obesidad general y abdominal (IMC y WC) y la enfermedad periodontal mostró una asociación significativa en el análisis de regresión logística, independiente de otros factores de confusión.

La investigación de Sede et al. (2014) ${ }^{(30)}$ buscó determinar la relación entre obesidad y estado periodontal de un grupo de pacientes nigerianos. Se estimó el índice de masa corporal (IMC) en $\mathrm{kg} / \mathrm{m}^{2}$ y se evaluó la salud gingival con el índice de sangrado al sondaje, la higiene oral (OHI-S) y la salud periodontal (BPE, de su sigla en inglés Basic Periodontal Examination). Los resultados del estudio fueron que la puntuación media de OHI-S fue de $2,16 \pm 1,13$ entre los participantes que tienen sobrepeso y $2,05 \pm 1,13$ entre los que no lo tienen $(p=0,543)$. El $75 \%$ de los individuos con un puntaje de $\mathrm{BPE}=0$, tenían un IMC normal, mientras que todos los participantes con el peor puntaje de BPE son obesos $(p=0,070)$. El estudio también reveló que el predictor probable de sangrado gingival es el IMC entre 35,0 y 39,9 (obesidad clase 2$)(p=0,046$; OR: 0,07; IC 95\% 0,01-0,96). Las conclusiones del estudio indican que no existe una relación estadísticamente significativa entre la obesidad y el estado periodontal. Sin embargo, el aumento del IMC puede ser un predictor de sangrado gingival.

Moura-Grec et al. (31) (2014) realizaron un metaanálisis sobre la asociación entre obesidad y periodontitis. Se escogieron estudios que investigaron si la obesidad es o no un factor 
de riesgo para la aparición de periodontitis. Identificaron 822 estudios, 31 cumplieron los criterios de inclusión y se revisaron para este trabajo. En 25 estudios el riesgo de periodontitis se asoció con obesidad o había una tendencia a ella. En seis estudios no se encontró ninguna relación. El metaanálisis mostró una asociación significativa entre la obesidad y la periodontitis (OR 1,30: IC $95 \%, 1,25-1,35$ ), y entre el IMC y la enfermedad periodontal (diferencia de medias: 2,75$)$. El estudio concluye que la obesidad se asocia con periodontitis, aunque los factores de riesgo que agravan estas enfermedades deben conocerse para entender mejor esta asociación. Para lograr la homogeneidad entre estudios se podría trabajar con muestras pareadas y evitar factores de confusión.

En 2015, Keller et al. ${ }^{(32)}$ revisaron sistemáticamente la asociación dependiente del tiempo entre la obesidad y la periodontitis y cómo los cambios de peso pueden afectar el desarrollo de periodontitis en la población general. Se buscó en estudios longitudinales y de intervención acerca de sobrepeso u obesidad y de exposición y periodontitis. Se analizaron ocho estudios longitudinales y cinco de intervención. En dos de las investigaciones longitudinales encontraron una asociación directa entre el grado de sobrepeso al inicio y el riesgo posterior de desarrollar periodontitis, y en otras tres observaron una asociación directa entre la obesidad y el desarrollo de periodontitis en adultos. Dos estudios de intervención sobre la influencia de la obesidad en los efectos del tratamiento periodontal mostraron que la respuesta a la terapia periodontal no quirúrgica fue mejor en los pacientes no obesos que en los obesos; los tres estudios restantes no informaron diferencias en el tratamiento periodontal entre los participantes obesos y no obesos. Uno de los estudios longitudinales fue ajustado para la proteína C-reactiva (PCR) y los marcadores biológicos de inflamación (IL-6 y FNT-a). Además, los marcadores de inflamación se analizaron por separado en tres de los cinco estudios de intervención. Los autores concluyeron que el sobrepeso, la obesidad, el aumento de peso pueden ser factores de riesgo para el desarrollo de periodontitis o el empeoramiento de las medidas periodontales.

En una revisión sistemática, Martínez-Herrera et al. (33) (2017) buscaron evidencia de la asociación entre obesidad y enfermedad periodontal para determinar los posibles mecanismos de esta relación. Se seleccionaron 284 artículos (ensayos clínicos controlados y estudios de observación que identificaron parámetros periodontales y de composición corporal). De ellos, 64 fueron preseleccionados y 28 se incluyeron en la revisión. Todos los estudios describieron una asociación entre obesidad y enfermedad periodontal, excepto dos. Aunque las causas de esta conexión aún no son claras, la resistencia a la insulina (como consecuencia de un estado inflamatorio crónico) y el estrés oxidativo podrían estar implicados en la relación entre obesidad y periodontitis. Los autores concluyeron que existe un patrón consistente de mayor riesgo de periodontitis en individuos con sobrepeso u obesidad.

En 2019, Santos et al. (34) evaluaron la asociación entre periodontitis severa con sobrepeso y obesidad. En una muestra aleatoria de 80 participantes obesos, 69 con sobrepeso y 87 individuos de peso normal se realizaron exámenes periodontales de boca completos y se utilizaron pruebas de regresión logística y chi-cuadrado para evaluar la asociación entre periodontitis severa y obesidad $(p \leq 0,05)$. Los resultados indican que la periodontitis grave se asoció significativamente con la obesidad (OR 3,25; IC 95\% 1,27-8, 31; $p=0,01)$, pero no con sobrepeso $(p=0,59)$, con lo que se concluye que existe una asociación entre los dos primeros cuadros. Se sugiere que los pacientes diagnosticados con obesidad sean referidos para una evaluación periodontal con más frecuencia.

Numerosos estudios han investigado la posible relación entre la enfermedad periodontal y la obesidad. Los resultados muestran que hay varios mecanismos por los que los adipocitos estimulan la producción de citocinas (FNT-a, leptina, IL-1 e IL-6) y de macrófagos (16,17). Las citocinas intervienen en el desarrollo y progresión de la enfermedad periodontal ${ }^{(18,19)}$; sin embargo, hasta el momento, no está clara esta asociación.

Una de las primeras investigaciones acerca de esta posible relación fue la de Dalla en $2005^{(22)}$, que determinó que la obesidad sería un posible factor de riesgo en el desarrollo de la enfermedad periodontal (OR 2,1: IC 95\% 1,1-3,9).

Luego otros autores investigaron esta asociación con resultados positivos en la mayoría de los casos. En el 2010, un metaanálisis de estudios epidemiológicos, realizado por Chaffee. et al ${ }^{(4)}$ encontró una asociación entre enfermedad periodontal y obesidad (OR 1,35: IC $95 \%$ 1,23-1,47). Por otro lado, Ylostalo et al., en 2011 (28), concluyen que la infección periodontal parece relacionarse, pero no encontró evidencias de causalidad por lo que enfatizó la necesidad de más estudios para aclarar el papel de la infección periodontal en la obesidad.

Se realizaron dos revisiones sistemáticas: una de Moura-Grec et al. ${ }^{(31)}$ que encontró que en 31 estudios que cumplieron con los criterios de inclusión, seis no presentaban ningún tipo de asociación, aunque existía una asociación significativa entre obesidad y periodontitis (OR = 1,30: IC 95\%, 1,25-1,35); y otra de Keller et al. (32) que un año después concluyó que el sobrepeso, la obesidad o el aumento de peso pueden ser factores de riesgo para el desarrollo de periodontitis o el empeoramiento de las medidas periodontales. En 2017, Martinez-Herrera et al. ${ }^{(33)}$ realizan una investigación basada en ensayos clínicos. Analizaron 284 estudios y seleccionaron 28 , en los que encontraron un patrón de riesgo más alto de periodontitis en individuos obesos. 
En estudios con mayor nivel de evidencia es concluyente que existe una posible asociación entre periodontitis y obesidad. Si bien la estadística muestra este hecho, el mecanismo fisiológico por el que se produce no es totalmente claro, lo que implica que se deben seguir investigando para determinar si la obesidad es un factor de riesgo de la enfermedad periodontal. En 2019, Santos et al. ${ }^{(34)}$ concluye que, si bien la periodontitis grave se asoció significativamente con la obesidad (OR 3,25, IC 95\% = 1,27-8,31; $p=0,01)$ no hubo una asociación con el sobrepeso $(p=0,59)$ (Tabla 1$)$.

Tabla 1. Estudios de la relación entre obesidad y enfermedad periodontal

\begin{tabular}{|c|c|c|c|c|c|}
\hline Autor & País & Objetivo & Muestra & Resultados & Conclusiones \\
\hline $\begin{array}{l}\text { Dalla et al. } \\
(2005)^{(22)}\end{array}$ & Brasil & $\begin{array}{l}\text { Evaluar la asociación } \\
\text { del sobrepeso y la } \\
\text { obesidad con la } \\
\text { periodontitis. }\end{array}$ & $\begin{array}{l}\text { Entrevistas y } \\
\text { exámenes clínicos } \\
\text { a } 706 \text { sujetos } \\
\text { entre } 30 \text { a } 65 \text { años } \\
\text { del sur de Brasil. }\end{array}$ & $\begin{array}{l}\text { Las mujeres obesas } \\
\text { tenían más } \\
\text { probabilidad de } \\
\text { tener periodontitis. } \\
\text { (OR 2,1: IC } 95 \% \text { : } \\
\text { 1,1-3,9). }\end{array}$ & $\begin{array}{l}\text { En adultos, la } \\
\text { obesidad (pero no el } \\
\text { sobrepeso) se asoció } \\
\text { significativamente } \\
\text { con la periodontitis. }\end{array}$ \\
\hline $\begin{array}{l}\text { Taghi et al. } \\
(2008)^{(23)}\end{array}$ & Irán & $\begin{array}{l}\text { Establecer la relación } \\
\text { entre enfermedad } \\
\text { periodontal, proteína } \\
\text { C-reactiva (PCR) y } \\
\text { obesidad. }\end{array}$ & $\begin{array}{l}\text { Estudiaron a } 166 \\
\text { pacientes ( } 35 \text { a } 59 \\
\text { años): } 83 \text { con } \\
\text { enfermedad } \\
\text { periodontal ( } 44 \\
\text { mujeres, } 39 \\
\text { hombres), y } 83 \\
\text { pacientes sanos. }\end{array}$ & $\begin{array}{l}\text { Obesidad: Mujeres: } \\
\text { OR 5,62 } \\
\text { (IC } 95 \%: 1,05-0,13 \text { ). } \\
\text { Hombres: OR 4,8 } \\
\text { (IC } 95 \% \text { : 0,65-35,19) } \\
\text { p } 0,05 .\end{array}$ & $\begin{array}{l}\text { La enfermedad } \\
\text { periodontal está } \\
\text { asociada con la } \\
\text { obesidad y con } \\
\text { elevación de la } \\
\text { PCR. }\end{array}$ \\
\hline $\begin{array}{l}\text { D'Aiuto et al. }^{(2008)^{(24)}}\end{array}$ & EE. UU. & $\begin{array}{l}\text { Evaluar la asociación } \\
\text { entre la periodontitis } \\
\text { y el síndrome } \\
\text { metabólico }\end{array}$ & $\begin{array}{l}\text { Análisis de datos } \\
\text { de la Third } \\
\text { National Health } \\
\text { and Nutrition. Se } \\
\text { estudió la } \\
\text { encuesta de } 13 \\
994 \text { hombres y } \\
\text { mujeres de } 17 \\
\text { años o más que se } \\
\text { realizaron un } \\
\text { examen } \\
\text { periodontal. }\end{array}$ & $\begin{array}{l}\text { Participantes } \geq \text { de } \\
45 \text { años con } \\
\text { periodontitis severas } \\
\text { eran } 2,31 \\
\text { (IC } 95 \% \text { : } \\
\text { 1,13 - 4,73) más } \\
\text { propensos a } \\
\text { síndrome } \\
\text { metabólico (SM) en } \\
\text { comparación de los } \\
\text { individuos sin } \\
\text { periodontitis } \\
\text { severas. La obesidad } \\
\text { no se asoció a la } \\
\text { periodontitis. } \\
\text { OR 1,54 } \\
\text { (IC } 95 \%: 0,99-2,40 \text { ). }\end{array}$ & $\begin{array}{l}\text { La periodontitis } \\
\text { severa se asocia con } \\
\text { el síndrome } \\
\text { metabólico en } \\
\text { individuos de } \\
\text { mediana edad. } \\
\text { No se pudo observar } \\
\text { una asociación entre } \\
\text { periodontitis y } \\
\text { obesidad. }\end{array}$ \\
\hline $\begin{array}{l}\text { Ylostalo et al. } \\
\text { (2008) (25) }\end{array}$ & Finlandia & $\begin{array}{l}\text { Examinar la asociación } \\
\text { del peso corporal } \\
\text { (IMC) con la infección } \\
\text { periodontal. }\end{array}$ & $\begin{array}{l}\text { Examen de salud } \\
\text { (Health 2000) a } \\
\text { nivel nacional. } \\
\text { Sujetos de } 30 \text { a } 49 \\
\text { años }(\mathrm{n}=2841) \text {. }\end{array}$ & $\begin{array}{l}\text { Se detectó una } \\
\text { asociación de } \\
\text { exposición- } \\
\text { respuesta del IMC } \\
\text { con periodontitis. }\end{array}$ & $\begin{array}{l}\text { Se evidenció una } \\
\text { asociación entre el } \\
\text { peso corporal y la } \\
\text { infección } \\
\text { periodontal entre la } \\
\text { población. }\end{array}$ \\
\hline
\end{tabular}




\begin{tabular}{|c|c|c|c|c|c|}
\hline Autor & País & Objetivo & Muestra & Resultados & Conclusiones \\
\hline $\begin{array}{l}\text { Khader et al. } \\
(2008)(26)\end{array}$ & Jordania & $\begin{array}{l}\text { Evaluar parámetros } \\
\text { periodontales entre } \\
\text { los pacientes con SM y } \\
\text { sin SM. }\end{array}$ & $\begin{array}{l}\text { Seleccionaron } 78 \\
\text { pacientes con SM } \\
\text { y } 78 \text { personas sin } \\
\text { SM para un } \\
\text { examen dental. } \\
\text { Todos los } \\
\text { pacientes con SM } \\
\text { presentaron } \\
\text { obesidad. }\end{array}$ & $\begin{array}{l}\text { La gravedad de la } \\
\text { enfermedad } \\
\text { periodontal fue } \\
\text { significativamente } \\
\text { mayor entre los } \\
\text { pacientes con SM en } \\
\text { comparación con el } \\
\text { grupo sin SM } \\
(p \leq 0,05) .\end{array}$ & $\begin{array}{l}\text { Los pacientes con } \\
\text { SM mostraron una } \\
\text { enfermedad } \\
\text { periodontal más } \\
\text { severa y extensa en } \\
\text { comparación con los } \\
\text { sujetos sin SM. }\end{array}$ \\
\hline $\begin{array}{l}\text { Khader et al. } \\
\text { (2009) }{ }^{(7)}\end{array}$ & Jordania & $\begin{array}{l}\text { Determinar la relación } \\
\text { entre periodontitis y } \\
\text { sobrepeso / obesidad } \\
\text { en la población } \\
\text { jordana. }\end{array}$ & $\begin{array}{l}\text { Examinaron a } 340 \\
\text { personas de entre } \\
18 \text { y } 70 \text { años que } \\
\text { acuden al centro } \\
\text { médico de la } \\
\text { Universidad de } \\
\text { Ciencia y } \\
\text { Tecnología de } \\
\text { Jordania. }\end{array}$ & $\begin{array}{l}\text { La obesidad } \\
\text { definida por el IMC } \\
\text { (OR) 2,9, (IC } 95 \% \text { : } \\
\text { 1,3; 6,1), WC alto } \\
\text { (OR 2,1, IC } 95 \% \text { : } \\
\text { 1,2; 3,7), y un } \\
\text { elevado porcentaje } \\
\text { de grasa (OR 1,8; } \\
\text { IC } 95 \% \text { : } 1,03 ; 3,3) \\
\text { permaneció } \\
\text { significativamente } \\
\text { asociado con } \\
\text { periodontitis. }\end{array}$ & $\begin{array}{l}\text { La obesidad medida } \\
\text { (IMC) y la } \\
\text { circunferencia de la } \\
\text { cintura alta, se } \\
\text { asociaron } \\
\text { significativamente } \\
\text { con el aumento de } \\
\text { probabilidad de } \\
\text { tener periodontitis. }\end{array}$ \\
\hline $\begin{array}{l}\text { Saxlin et al. } \\
(2010)(27)\end{array}$ & Finlandia & $\begin{array}{l}\text { Investigar la } \\
\text { asociación entre el } \\
\text { peso corporal y la } \\
\text { infección periodontal } \\
\text { en un contexto } \\
\text { longitudinal. }\end{array}$ & $\begin{array}{l}\text { Estudio basado en } \\
\text { una subpoblación } \\
\text { de la Encuesta de } \\
\text { Salud } 2000 \text {. } \\
\text { Incluyó sujetos } \\
\text { dentados (de } 30 \text { a } \\
59 \text { años) que } \\
\text { habían participado } \\
\text { en el seguimiento } \\
\text { sobre la salud } \\
\text { bucal de los } \\
\text { adultos } \\
\text { finlandeses. Se } \\
\text { realizó } \\
\text { aproximadamente } \\
4 \text { años después } \\
\text { ( } n=5396 \text { ). }\end{array}$ & $\begin{array}{l}\text { La asociación del } \\
\text { peso corporal y el } \\
\text { número de dientes } \\
\text { con periodontitis } \\
\text { fue débil y no tuvo } \\
\text { significación } \\
\text { estadística RR } 1,1 \\
\text { (IC } 95 \% \text { : 0,8-1,7). }\end{array}$ & $\begin{array}{l}\text { No hay evidencia de } \\
\text { que el sobrepeso y } \\
\text { la obesidad puedan } \\
\text { considerarse } \\
\text { factores de riesgo } \\
\text { significativos en la } \\
\text { patogénesis de la } \\
\text { infección } \\
\text { periodontal. }\end{array}$ \\
\hline
\end{tabular}




\begin{tabular}{|c|c|c|c|c|c|}
\hline Autor & País & Objetivo & Muestra & Resultados & Conclusiones \\
\hline $\begin{array}{l}\text { Chaffee et al. } \\
\qquad(2010)^{(4)}\end{array}$ & EE. UU. & $\begin{array}{l}\text { Recopilar de manera } \\
\text { sistemática la } \\
\text { evidencia de una } \\
\text { relación } \\
\text { obesidad-enfermedad } \\
\text { periodontal a partir } \\
\text { de estudios previos. }\end{array}$ & $\begin{array}{l}\text { Incluyó } 554 \text { citas, } \\
70 \text { estudios que } \\
\text { cumplieron los } \\
\text { criterios de } \\
\text { inclusión y que } \\
\text { representan } 57 \\
\text { poblaciones } \\
\text { independientes. }\end{array}$ & $\begin{array}{l}\text { En } 41 \text { estudios se } \\
\text { sugiere una } \\
\text { asociación positiva.El } \\
\text { OR fue de } 1,35 \\
\text { (IC } 95 \%: 1,23 \text { a } \\
\text { 1,47), con alguna } \\
\text { evidencia de una } \\
\text { asociación más } \\
\text { fuerte entre adultos } \\
\text { más jóvenes y } \\
\text { mujeres. }\end{array}$ & $\begin{array}{l}\text { La asociación } \\
\text { positiva fue } \\
\text { consistente y } \\
\text { coherente con un } \\
\text { papel } \\
\text { biológicamente } \\
\text { plausible para la } \\
\text { obesidad en el } \\
\text { desarrollo de la } \\
\text { enfermedad } \\
\text { periodontal. }\end{array}$ \\
\hline $\begin{array}{l}\text { Saxlin et al. } \\
\qquad(2011)^{(28)}\end{array}$ & Finlandia & $\begin{array}{l}\text { Investigar el rol de la } \\
\text { infección periodontal } \\
\text { en la obesidad en una } \\
\text { población adulta. }\end{array}$ & $\begin{array}{l}\text { Examen de salud } \\
\text { (Health 2000) a } \\
\text { nivel nacional. } \\
\text { Sujetos de } 30 \text { a } 49 \\
\text { años. No } \\
\text { diabéticos } \\
\text { ( } n=2784 \text { ). }\end{array}$ & $\begin{array}{l}\text { Se encontró que el } \\
\text { número de dientes } \\
\text { con bolsas } \\
\text { periodontales } \\
\text { profundas estaba } \\
\text { asociado con el IMC. } \\
\text { OR 2,4 (IC } 95 \% \text { : } \\
\text { 1,5-3,8). }\end{array}$ & $\begin{array}{l}\text { La infección } \\
\text { periodontal parece } \\
\text { estar asociada con } \\
\text { la obesidad. Sin } \\
\text { embargo, no hay } \\
\text { inferencias acerca } \\
\text { de la causalidad. }\end{array}$ \\
\hline $\begin{array}{l}\text { Palle et al. } \\
(2013)^{(29)}\end{array}$ & India & $\begin{array}{l}\text { Correlacionar la } \\
\text { obesidad (IMC), la } \\
\text { circunferencia de la } \\
\text { cintura (WC) y los } \\
\text { parámetros de la } \\
\text { enfermedad } \\
\text { periodontal. }\end{array}$ & $\begin{array}{l}\text { Se examinaron } 201 \\
\text { pacientes y se } \\
\text { incluyeron a } \\
\text { quienes tenían } \\
\text { antecedentes de } \\
\text { enfermedades } \\
\text { cardiovasculares y } \\
\text { en tratamiento. }\end{array}$ & $\begin{array}{l}\text { Observaron una } \\
\text { asociación } \\
\text { significativa entre } \\
\text { el IMC y la gravedad } \\
\text { de periodontitis } \\
(p<0,001) \text {, el IMC y } \\
\text { la severidad de la } \\
\text { periodontitis } \\
(p<0,001) \text {, y el WC } \\
\text { y la severidad de la } \\
\text { periodontitis } \\
(p<0,001) .\end{array}$ & $\begin{array}{l}\text { La relación entre las } \\
\text { medidas de obesidad } \\
\text { general y abdominal } \\
\text { (IMC y WC) y la } \\
\text { enfermedad } \\
\text { periodontal mostró } \\
\text { una asociación } \\
\text { significativa } \\
\text { independiente de } \\
\text { otros factores de } \\
\text { confusión. }\end{array}$ \\
\hline $\begin{array}{l}\text { Sede et al. } \\
(2014)^{(30)}\end{array}$ & Nigeria & $\begin{array}{l}\text { Determinar la relación } \\
\text { entre obesidad y } \\
\text { estado periodontal de } \\
\text { un grupo de pacientes } \\
\text { nigerianos. }\end{array}$ & $\begin{array}{l}\text { Participaron } 156 \\
\text { pacientes. Se } \\
\text { estimó el (IMC) y } \\
\text { se evaluó la salud } \\
\text { periodontal (BPE). }\end{array}$ & $\begin{array}{l}\text { El } 75 \% \text { de los } \\
\text { individuos con un } \\
\text { puntaje de BPE de } 0 \\
\text { tenían un IMC } \\
\text { normal. Todos los } \\
\text { participantes con el } \\
\text { peor puntaje de BPE } \\
\text { pertenecen a } \\
\text { individuos obesos } \\
(p=0,070) .\end{array}$ & $\begin{array}{l}\text { No existe una } \\
\text { relación } \\
\text { estadísticamente } \\
\text { significativa entre la } \\
\text { obesidad y el estado } \\
\text { periodontal. }\end{array}$ \\
\hline
\end{tabular}




\begin{tabular}{|c|c|c|c|c|c|}
\hline Autor & País & Objetivo & Muestra & Resultados & Conclusiones \\
\hline $\begin{array}{l}\text { Moura et al. } \\
\qquad(2014)^{(31)}\end{array}$ & Brasil & $\begin{array}{l}\text { Metaanálisis de los } \\
\text { estudios sobre la } \\
\text { asociación entre } \\
\text { obesidad y } \\
\text { periodontitis. }\end{array}$ & $\begin{array}{l}\text { Identificaron } 822 \\
\text { estudios y } 31 \text { se } \\
\text { incluyeron en esta } \\
\text { revisión. }\end{array}$ & $\begin{array}{l}\text { Los resultados del } \\
\text { estudio indicaron } \\
\text { que el riesgo de } \\
\text { periodontitis se } \\
\text { asoció con obesidad } \\
\text { en } 25 \text { estudios, } \\
\text { aunque no se asoció } \\
\text { en seis estudios. El } \\
\text { metaanálisis mostró } \\
\text { una asociación } \\
\text { significativa con la } \\
\text { obesidad y la } \\
\text { periodontitis } \\
\text { (OR } 1,30: \text { IC } 95 \% \text {, } \\
1,25 \text { - } 1,35)\end{array}$ & $\begin{array}{l}\text { La obesidad se } \\
\text { asoció con } \\
\text { periodontitis. Es } \\
\text { necesario identificar } \\
\text { los factores de } \\
\text { riesgo que agravan } \\
\text { estas enfermedades } \\
\text { para entender mejor } \\
\text { esta asociación. }\end{array}$ \\
\hline $\begin{array}{l}\text { Keller et al. } \\
(2015)^{(32)}\end{array}$ & Dinamarca & $\begin{array}{l}\text { Examinar la asociación } \\
\text { entre obesidad y } \\
\text { periodontitis y cómo } \\
\text { los cambios de peso } \\
\text { pueden afectar la } \\
\text { evolución de } \\
\text { periodontitis en la } \\
\text { población general. }\end{array}$ & $\begin{array}{l}\text { Se incluyeron } 13 \\
\text { artículos. La } \\
\text { búsqueda } \\
\text { bibliográfica se } \\
\text { realizó entre } \\
\text { diciembre de } 2013 \\
\text { y junio de } 2014 \text { en } \\
\text { Pubmed/ Medline. }\end{array}$ & $\begin{array}{l}\text { Dos de los estudios } \\
\text { encontraron una } \\
\text { asociación directa } \\
\text { entre el sobrepeso y } \\
\text { el riesgo de } \\
\text { desarrollar } \\
\text { periodontitis, y tres } \\
\text { estudios mostraron } \\
\text { una asociación } \\
\text { directa entre } \\
\text { obesidad y el } \\
\text { desarrollo de } \\
\text { periodontitis en } \\
\text { adultos. }\end{array}$ & $\begin{array}{l}\text { Esta revisión } \\
\text { sistemática sugiere } \\
\text { que el sobrepeso, la } \\
\text { obesidad, el } \\
\text { aumento de peso y } \\
\text { el aumento de la } \\
\text { circunferencia de la } \\
\text { cintura pueden ser } \\
\text { factores de riesgo } \\
\text { para el desarrollo o } \\
\text { empeoramiento de } \\
\text { los cuadros } \\
\text { periodontales. }\end{array}$ \\
\hline $\begin{array}{l}\text { Martínez et al. } \\
\qquad(2017)^{(33)}\end{array}$ & España & $\begin{array}{l}\text { Revisar } \\
\text { sistemáticamente la } \\
\text { asociación entre la } \\
\text { obesidad y la } \\
\text { enfermedad } \\
\text { periodontal. }\end{array}$ & $\begin{array}{l}\text { Se recopilaron } 284 \\
\text { artículos De ellos, } \\
64 \text { fueron } \\
\text { preseleccionados, } \\
\text { y } 28 \text { se incluyeron } \\
\text { en la revisión. }\end{array}$ & $\begin{array}{l}\text { Todos los estudios } \\
\text { describieron una } \\
\text { asociación entre } \\
\text { obesidad y } \\
\text { enfermedad } \\
\text { periodontal, } \\
\text { excepto dos } \\
\text { artículos. }\end{array}$ & $\begin{array}{l}\text { La asociación entre } \\
\text { obesidad y } \\
\text { periodontitis fue } \\
\text { consistente con un } \\
\text { patrón que } \\
\text { establece que existe } \\
\text { mayor riesgo de } \\
\text { periodontitis en los } \\
\text { individuos con } \\
\text { sobrepeso u } \\
\text { obesidad. }\end{array}$ \\
\hline
\end{tabular}




\begin{tabular}{|c|c|c|c|c|c|}
\hline Autor & País & Objetivo & Muestra & Resultados & Conclusiones \\
\hline $\begin{array}{l}\text { Santos et al. } \\
\qquad(2019)^{(34)}\end{array}$ & Brasil & $\begin{array}{l}\text { Evaluar la asociación } \\
\text { de periodontitis } \\
\text { severa con sobrepeso } \\
\text { y obesidad. }\end{array}$ & $\begin{array}{l}\text { Intervinieron } 80 \\
\text { participantes } \\
\text { obesos, } 69 \text { con } \\
\text { sobrepeso y } 87 \\
\text { individuos de peso } \\
\text { normal }\end{array}$ & $\begin{array}{l}\text { La periodontitis } \\
\text { grave se asoció } \\
\text { significativamente } \\
\text { con la obesidad } \\
(\text { OR } 3.25, \text { IC } 95 \% \\
1,27-8,31 ; p=0,01) \\
\text { pero no con } \\
\text { sobrepeso }(p=0,59) \text {. }\end{array}$ & $\begin{array}{l}\text { La periodontitis } \\
\text { grave se asoció con } \\
\text { obesidad, pero no } \\
\text { con sobrepeso. }\end{array}$ \\
\hline
\end{tabular}

Estos datos resultan relevantes porque de acuerdo a la evidencia se puede determinar que, en un estadio más avanzado de obesidad, la enfermedad periodontal es más significativa en comparación a las personas con peso normal. La información disponible indica que el mecanismo de acción de las citocinas estaría relacionado con la presencia de células inflamatorias en pacientes obesos. Además, es necesario considerar los factores de riesgo asociados a enfermedades sistémicas que desencadenan una respuesta de los mediadores de la inflamación y que afectan nuestro organismo.

\section{CONCLUSIONES}

Es posible determinar, con base en la evidencia disponible, la probabilidad de que exista relación entre enfermedad periodontal y obesidad. Sugerimos realizar más estudios con mayor grado de evidencia y de rigor científico para confirmar esta posible asociación. La implementación y renovación de los enfoques de programas de salud oral deben estar orientados al diagnóstico y reducción de factores de riesgo de enfermedades inflamatorias.

Contribuciones de los autores: Jorge Girano Castaños realizó el análisis, revisión, selección y redacción del artículo de revisión. José Robello Malatto realizó el análisis, revisión y evaluación crítica del artículo de revisión.

Fuentes de financiamiento: Este artículo ha sido financiado por los autores.

Conflictos de interés: Los autores declaran no tener ningún conflicto de interés.

\section{REFERENCIAS BIBLIOGRÁFICAS}

1. Organización Mundial de la Salud. Obesidad y sobrepeso [internet]. 2012. Disponible en: https://www.who.int/es/newsroom/fact-sheets/detail/obesity-and-overweight

2. Ogden CL, Carroll MD, Kit BK, Flegal KM. Prevalence of obesity in the United States, 2009-2010. NCHS Data Brief. 2012; (82): 1-8.
3. Álvarez-Dongo D, Sánchez-Abanto J, Gómez-Guizado G. Sobrepeso y obesidad: prevalencia y determinantes sociales del exceso de peso en la población peruana (2009-2010). Rev Perú Med Exp Salud Pública. 2012; 29(3): 303-13.

4. Chaffee BW, Weston SJ. Association between chronic periodontal disease and obesity: a systematic review and meta-analysis. J Periodontol. 2010; 81(12): 1708-24.

5. Organización Mundial de la Salud del Perú. Salud bucodental [internet]. 2007. Disponible en: https://www.who.int/es/newsroom/fact-sheets/detail/oral-health

6. Ministerio de Salud del Perú. Salud bucal [Internet]. Disponible en: http://www.minsa.gob.pe/portada/est_san/saludbucal.htm

7. Khader YS, Bawadi HA, Haroun TF, Alomari M, Tayyem RF. The association between periodontal disease and obesity among adults in Jordan. J Clin Periodontol. 2009; 36(1): 18-24.

8. Dennison EM, Syddall HE, Sayer AA, Martin HJ, Cooper C. Lipid profile, obesity and bone mineral density: the hertfordshire cohort study. QJM. 2007; 100(5): 297-303.

9. Kim EJ, Jin BH, Bae KH. Periodontitis and obesity: a study of the fourth Korean National Health and nutrition examination survey. J Periodontol. 2011; 82(4): 533-42.

10. Gürgan CA, Altay U, Ağbaht K. Changes in inflammatory and metabolic parameters after periodontal treatment in obese and non-obese patients. J Periodontol. 2013; 84(1): 13- 23.

11. World Health Organization. Technical report series 894: Obesity: preventing and managing the global epidemic [Internet]. Geneva: World Health Organization. 2000.

12. Bartold PM, Cantley MD, Haynes DR. Mechanisms and control of pathologic bone loss in periodontitis. Periodontol. 2010; 53: 55-69.

13. Marchetti E, Monaco A, Procaccini L, Mummolo S, Gatto R, Tetè S, et al. Periodontal disease: the influence of metabolic syndrome. Nutr Metab (Lond). 2012; 9: 88.

14. Trayhurn P, Wood IS. Adipokines: inflammation and the pleiotropic role of white adipose tissue. Br J Nutr. 2004; 92(3): 347-55.

15. Ouchi N, Kihara S, Funahashi T, Nakamura T, Nishida M, Kumada $M$, et al. Reciprocal association of C-reactive protein with adiponectin in blood stream and adipose tissue. Circulation. 2003; 107(5): 671-4.

16. Simons PJ, van den Pangaart PS, van Roomen CP, Aerts JM, Boon L. Cytokine-mediated modulation of leptin and adiponectin secretion during in vitro adipogenesis: evidence that tumor necrosis factoralpha- and interleukin-1beta-treated human preadipocytes are potent leptin producers. Cytokine. 2005; 32(2): 94-103.

17. Cancello R, Clément K. Is obesity an inflammatory illness? Role of low-grade inflammation and macrophage infiltration in human white adipose tissue. BJOG. 2006; 113(10): 1141-7. 
18. Karlsson EA, Beck MA. The burden of obesity on infectious disease. Exp Biol Med (Maywood). 2010; 235(12): 1412-24.

19. Reynolds MA. Modifiable risk factors in periodontitis: at the intersection of aging and disease. Periodontol 2000. 2014; 64(1): 7-19.

20. Dahiya P, Kamal R, Gupta R. Obesity, periodontal and general health: relationship and management. Indian J Endocr Metab. 2012; 16(1): 88-93.

21. Endo $Y$, Tomofuji T, Ekuni D, Irie K, Azuma T, Tamaki N, et al. Experimental periodontitis induces gene expression of proinflammatory cytokines in liver and white adipose tissues in obesity. J Periodontol. 2010; 81(4): 520-6.

22. Dalla Vecchia CF, Susin C, Kuchenbecker Rosing C, Oppermann $\mathrm{RV}$, Albandar JM. Overweight and obesity as risk indicators for periodontitis in adults. J Periodontol. 2005; 76(10): 1721-8.

23. Taghi Chitsazi M, Pourabbas R, Shirmohammadi A, Ahmadi Zenouz $G$, Hossein Vatankhah A. Association of periodontal diseases with elevation of serum C-reactive protein and body mass index. J Dent Res Clin Dent Prospects. 2008; 2(1): 9-14.

24. D'Aiuto F, Sabbah W, Netuveli G, Donos N, Hingorani AD, Deanfield $J$, et al. Association of the metabolic syndrome with severe periodontitis in a large U.S. population-based survey. J Clin Endocrinol Metab. 2008; 93(10): 3989-94.

25. Ylostalo P, Suominen-Taipale L, Reunanen A, Knuuttila M. Association between body weight and periodontal infection. J Clin Periodontol. 2008; 35(4): 297-304.

26. Khader Y, Khassawneh B, Obeidat B, Hammad M, El-Salem K, Bawadi $\mathrm{H}$, et al. Periodontal status of patients with metabolic syndrome compared to those without metabolic syndrome. J Periodontol. 2008; 79(11): 2048-53.

27. Saxlin T, Ylostalo P, Suominen-Taipale L, Aromaa A, Knuuttila M. Overweight and obesity weakly predict the development of periodontal infection. J Clin Periodontol. 2010; 37(12): 1059-67.

28. Saxlin T, Ylostalo P, Suominen-Taipale L, Mannisto S, Knuuttila M. Association between periodontal infection and obesity: results of the health 2000 survey. J Clin Periodontol. 2011; 38(3): 236-42

29. Palle AR, Reddy CM, Shankar BS, Gelli V, Sudhakar J, Reddy KK. Association between obesity and chronic periodontitis: a crosssectional study. J Contemp Dent Pract. 2013; 14(2): 168-73.

30. Sede MA, Ehizele AO. Relationship between obesity and oral diseases. Niger J Clin Pract. 2014; 17(6): 683-90.

31. Moura-Grec PG, Marsicano JA, Paz de Carvalho CA, Sales-Peres SH. Obesity and periodontitis: systematic review and meta-analysis. Cien Saude Colet. 2014; 19(6): 1763-72.

32. Keller A, Rohde JF, Raymond K, Heitmann BL. Association between periodontal disease and overweight and obesity: a systematic review. J Periodontol. 2015; 86(6): 766-76.

33. Martinez-Herrera M, Silvestre-Rangil J, Silvestre F-J. Association between obesity and periodontal disease. A systematic review of epidemiological studies and controlled clinical trials. Med Oral Patol Oral Cir Bucal. 2017; 22(6): 708-15.

34. Santos T, Ramos Cury P, Santos E, Vasconcelos R, Dos Santos JN, Pedreira Ramalho LM. Association between severe periodontitis and obesity degree: a preliminary study. Oral Health Prev Dent. 2019; 17(2): 173-7.

\section{Correspondencia:}

Jorge Girano Castaños

Dirección: Calle Torrigiano Pietro. 492. San Borja. Lima, Perú. Teléfono: 951726380

Correo electrónico: jorgegirano@gmail.com

Recibido: 29 de octubre de 2019

Evaluado: 08 de enero de 2020

Aprobado: 21 de enero de 2020

(c) La revista. Publicado por Universidad de San Martín de Porres, Perú (cc) BY Licencia de Creative Commons Artículo en acceso abierto bajo términos de Licencia Creative Commons Atribución 4.0 Internacional. (http://creativecommons.org/licenses/by/4.0/)

\section{ORCID iDs}

Jorge Girano Castaños

(1) https: / / orcid.org/0000-0003-1854-5001

José Robello Malatto

가 https: / / orcid.org/0000-0003-3942-0320 